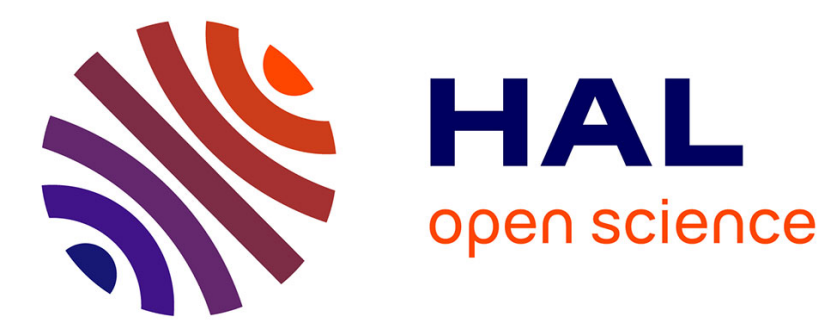

\title{
Simultaneous Quantification of Indium and Nitrogen Concentration in InGaNAs Using HAADF-STEM
}

Tim Grieb, Knut Müller, Emmanuel Cadel, Andreas Beyer, Marco Schowalter, Etienne Talbot, Kerstin Volz, Andreas Rosenauer

\section{- To cite this version:}

Tim Grieb, Knut Müller, Emmanuel Cadel, Andreas Beyer, Marco Schowalter, et al.. Simultaneous Quantification of Indium and Nitrogen Concentration in InGaNAs Using HAADF-STEM. Microscopy and Microanalysis, 2014, 20 (06), pp.1740 - 1752. 10.1017/S1431927614013051 . hal-01633486

\section{HAL Id: hal-01633486 https://hal.science/hal-01633486}

Submitted on 16 Jan 2019

HAL is a multi-disciplinary open access archive for the deposit and dissemination of scientific research documents, whether they are published or not. The documents may come from teaching and research institutions in France or abroad, or from public or private research centers.
L'archive ouverte pluridisciplinaire HAL, est destinée au dépôt et à la diffusion de documents scientifiques de niveau recherche, publiés ou non, émanant des établissements d'enseignement et de recherche français ou étrangers, des laboratoires publics ou privés. 


\title{
Simultaneous Quantification of Indium and Nitrogen Concentration in InGaNAs Using HAADF-STEM
}

\author{
Tim Grieb, ${ }^{1, *}$ Knut Müller, ${ }^{1}$ Emmanuel Cadel, ${ }^{2}$ Andreas Beyer, ${ }^{3}$ Marco Schowalter, ${ }^{1}$ \\ Etienne Talbot, ${ }^{2}$ Kerstin Volz, ${ }^{3}$ and Andreas Rosenauer ${ }^{1}$ \\ ${ }^{1}$ Institute of Solid State Physics, University of Bremen, Otto-Hahn-Allee 1, 28359 Bremen, Germany \\ ${ }^{2}$ Groupe de Physique des Matériaux (GPM) UMR 6634, Normandie Université, Université et INSA de RouenCNRS, Av. de \\ l'Université, BP 12, 76801 Saint Etienne du Rouvray, France \\ ${ }^{3}$ Materials Science Center and Faculty of Physics, Philipps University Marburg, Hans Meerwein Straße, 35032 Marburg, \\ Germany
}

\begin{abstract}
To unambiguously evaluate the indium and nitrogen concentrations in $\operatorname{In}_{x} \mathrm{Ga}_{1-x} \mathrm{~N}_{y} \mathrm{As}_{1-y}$, two independent sources of information must be obtained experimentally. Based on high-resolution scanning transmission electron microscopy (STEM) images taken with a high-angle annular dark-field (HAADF) detector the strain state of the InGaNAs quantum well is determined as well as its characteristic HAADF-scattering intensity. The strain state is evaluated by applying elasticity theory and the HAADF intensity is used for a comparison with multislice simulations. The combination of both allows for determination of the chemical composition where the results are in accordance with X-ray diffraction measurements, three-dimensional atom probe tomography, and further transmission electron microscopy analysis. The HAADF-STEM evaluation was used to investigate the influence of As-stabilized annealing on the InGaNAs/GaAs sample. Photoluminescence measurements show an annealing-induced blue shift of the emission wavelength. The chemical analysis precludes an elemental diffusion as origin of the energy shift-instead the results are in agreement with a model based on an annealing-induced redistribution of the atomic next-neighbor configuration.
\end{abstract}

\section{INTRODUCTION}

As even small amounts of incorporated nitrogen drastically lower the band gap of the III-V semiconductor $\operatorname{In}_{x} \mathrm{Ga}_{1-x}$ $\mathrm{N}_{\mathrm{y}} \mathrm{As}_{1-y}$, the material is of high technological interest for the design of laser diodes with emission wavelengths of 1.3 or $1.55 \mu \mathrm{m}$, which are called telecom windows in glass-fiber guidance (Kondow et al., 1997; Riechert et al., 2002). A second application is the incorporation of InGaNAs into multijunction solar cells (see, e.g., Friedman et al., 1998; Volz et al., 2004).

Usually the epitaxially grown structures are annealed thermally in order to increase the optical efficiency. This annealing process is attended by an increase of the band-gap energy, or a blue shift of the emission wavelength. Different annealing-induced structural changes have been reported, such as indium homogenization (Buyanova et al., 2000; Kitatani et al., 2000; Albrecht et al., 2002), diffusion of nitrogen (out of the quantum well; Spruytte et al., 2001) or toward the quantum-well interfaces (Litvinov et al., 2004), or changing of the atomic next-neighbor correlation (Klar et al., 2001; Kurtz et al., 2001; Volz et al., 2008). For technological purposes it is important to understand the origin of the blue shift, so it is necessary to achieve quantitative information from the structures on the atomic scale.
For materials with only one unknown chemical concentration, many methods exist to perform quantitative analyses: X-ray diffraction (XRD) is a common tool to investigate bulk materials. For small structures such as quantum wells, transmission electron microscopy (TEM) offers further methods: strain state analysis (SSA) allows for calculation of the chemical concentration from the measured local strain. A special feature of zincblende crystals is the chemical sensitivity of the 002-beam intensity, which can be used for quantitative analyses (e.g., Rosenauer et al., 1998).

Investigation of the quaternary material $\operatorname{In}_{x} \mathrm{Ga}_{1-x} \mathrm{~N}_{y} \mathrm{As}_{1-y}$ is complex: most signals, such as the local strain or the 002-beam intensity in TEM depend on both compositions, $x$ and $y$. An unambiguous evaluation is therefore not possible with a single method and without further information.

Effort was made to develop methods for evaluation of the indium and the nitrogen concentration in InGaNAs. Grillo et al. (2001) combined the information of local strain measured from a high-resolution TEM image and the chemically sensitive 002 intensity measured from a dark-field TEM image. Müller et al. (2011) presented the 3-beam method that evaluates a single TEM lattice-fringe image from the interference of the 000,002 , and 022 beams using a special aperture.

TEM analysis can be supported by spectroscopy methods as electron energy-loss spectroscopy or energy-dispersive $\mathrm{X}$-ray spectroscopy. However, in the case of InGaNAs, both methods suffer from the fact that small amounts of nitrogen $(1-2 \%)$ are hardly measurable in a quantitative way. 
The quantification method presented in this paper is based on the evaluation of high-angle annular dark-field (HAADF) intensity in scanning transmission electron microscopy (STEM) by a comparison of experimental intensities with simulated ones. The application of this technique to perform compositional mapping was already shown for ternary materials, e.g., for zincblende InGaAs (Carlino, 2010), GaNAs (Grieb et al., 2012), InAsP (Molina et al., 2009), and for wurtzite AlGaN (Rosenauer et al., 2009) and InGaN (Rosenauer et al., 2011).

In order to simultaneously analyze the indium and the nitrogen concentration, a SSA from high-resolution scanning transmission electron microscopy (HRSTEM) images is combined with an evaluation of the HAADF intensity from the same image. It has already been shown that even for a large specimen thickness SSA of HRSTEM images is an adequate tool for quantitative evaluations (Grieb et al., 2012). Determining the strain state directly from HRSTEM images has the advantage that both types of information, strain and HAADF intensity, can be obtained from the same image and therefore from the same specimen position.

To evaluate strain state, the elasticity theory is applied. The HAADF intensity is then evaluated by a comparison with simulations based on the multislice algorithm. It was shown, e.g., by Grillo et al. (2008), Glas (2004), and Müller et al. (2010) that the influence of static atomic displacements (SADs) related to In or $\mathrm{N}$ atoms has a large influence on the scattering intensity of (In) $\mathrm{Ga}(\mathrm{N})$ As. SADs are local distortions of the ideal atomic lattice, here caused by the small covalent radius of nitrogen compared with arsenic and the large covalent radius of indium compared with gallium (Glas, 2004). It was also shown that SADs play an important role also in HAADF-STEM of ternary dilute GaNAs, containing $<5 \%$ nitrogen (Grieb et al., 2012)). For this reason, SADs were taken into account in the HAADF-intensity simulations for InGaNAs presented in this paper.

\section{Materials and Methods}

\section{Specimen Preparation}

Two types of InGaNAs/GaAs quantum-well samples were investigated. The sample with a nominal indium concentration of $8 \%$ will be named sample $\mathrm{A}$ in the following, the sample with a higher indium content of $31 \%$ sample B.

The zincblende InGaNAs/GaAs samples were grown in the [001] direction by metal organic vapor phase epitaxy (MOVPE) in a horizontal AIX 200 GFR reactor. The nominal values of the indium and nitrogen concentrations were determined from high-resoluion X-ray diffraction (HRXRD) measurements. The nominal thicknesses of the quaternary layer was $8.5 \mathrm{~nm}$ in sample B with a GaAs-capping layer of $130 \mathrm{~nm}$. The investigated layer in sample A with a nominal thickness of $15 \mathrm{~nm}$ was part of a multilayer structure. The layer was separated by 100-nm GaAs barriers to both sides.

Rocking curves $(\omega-2 \theta)$ of the 004 reflection were acquired using an X'Pert Pro PANalytical diffractometer.
The profiles were fitted by fixing the indium concentrations. The amount of indium was derived from HRXRD investigations of ternary InGaAs samples grown under identical growth conditions. The nitrogen content was derived to be 3\% (sample A) and 1.5\% (sample B).

For sample B, two specimens were investigated where one remained without temperature treatment (named as-grown), the second one (identically grown) was annealed for $60 \mathrm{~min}$ at $725^{\circ} \mathrm{C}$ in the MOVPE reactor under stabilizing As atmosphere (named annealed) to investigate the effects of annealing. For details of the annealing procedure see Klar et al. (2001). Room temperature PL measurements were performed on both samples.

All TEM lamellae were prepared using the focused ion beam (FIB) system FEI Nova Nanolab 200 and subsequently milled at $400 \mathrm{~V}$ in a Technoorg Linda Gentle Mill IV5 to reduce the amorphous surface layer occurring during FIB preparation (Scott et al., 2006; Mehrtens et al., 2012). Each lamella was plasma cleaned shortly before TEM investigations, using a Binder TPS 216 plasma cleaner operated with Ar/O plasma. The plasma cleaning reduced the effect of electron-beam deposition of parasitic carbohydrates on the specimen surface.

\section{Instruments}

We used an FEI Titan 80/300 microscope at an acceleration voltage of $300 \mathrm{kV}$. The measured semi-convergence angle in STEM was $8 \mathrm{mrad}$ and the spherical aberration constant of the microscope was $1.2 \mathrm{~mm}$. The gun-lens setting was 6 , the spot size 9 , and the extraction voltage $4.5 \mathrm{kV}$. See Rosenauer et al. $(2009,2011)$ for further details on the STEM measurements.

A Fischione 3000 HAADF detector was used at a camera length of $196 \mathrm{~mm}$, which corresponds to a range of detection angles between 36 and $230 \mathrm{mrad}$ (Grieb et al., 2012).

For TEM analysis, $C_{S^{-}}$corrected images were taken with microscope settings presented by Müller et al. (2011). All (S)TEM measurements were performed at room temperature.

The Titan 80/300 microscope allows collection of an image of the HAADF detector simply by switching into the imaging mode. This detector scan shown in Figure 1a directly visualizes the local sensitivity of the detector. Owing to the obvious nonuniform sensitivity of the HAADF detector, whose sensitive area deviates from a uniform ring, the local sensitivity obtained from the detector scan has to be taken into account when simulating HAADF intensities (Rosenauer et al., 2009). For the STEM measurements, the central beam was positioned at the center of the HAADF detector as described by LeBeau \& Stemmer (2008) in more detail.

The detector scan also provides information on the total probe intensity. For normalization of the STEM images, the mean intensity on the detector $I_{\text {det }}$ and the mean intensity in the vacuum $I_{\mathrm{vac}}$ can be derived from the detector scan (see Fig. 1). The value of $I_{\mathrm{vac}}$ is not 0 owing to an offset of the amplifier. To provide a linear signal transfer of the amplifier, contrast and brightness were adjusted so that $I_{\text {det }}$ was $\sim 4 \times 10^{4}$ and $I_{\mathrm{vac}} \sim 1 \times 10^{4}$ (Rosenauer et al., 2009). 
Sample B was investigated with the three-dimensional (3D) atom probe on a laser-assisted 3DAP CamecaLaWaTAP. The analysis was performed at $5 \times 10^{-10}$ Torr rest pressure and a specimen temperature fixed at $80 \mathrm{~K}$. The laser system is an amplified ytterbium-doped laser (Amplitude system s-pulse, $\lambda=1,030 \mathrm{~nm}$ ). An $~ 50 \mathrm{~nJ} / 450 \mathrm{fs}$ pulse focused onto an $\sim 0.1 \mathrm{~mm}^{2}$ spot is used to obtain a $\mathrm{Ga} /$ As ratio close to 1 in the GaAs substrate. Preliminary testing results confirm that the use of infrared laser pulses, instead of blue- and green-laser pulses, lead to a homogeneous distribution of gallium atoms within the 3DAP reconstructed volumes. A shank angle-based method was used to reconstruct the 3DAP data. This method uses simple geometric considerations that give the change in radius of curvature as a function of the depth and shank angle. For a given atomic density and detection efficiency, the reconstruction parameters were the initial radius of curvature and the shank angle (the specimen shape was obtained from scanning electron micrographs). By adjusting these parameters, the In-layer thickness was adjusted to match with the HRSTEM image of the InGaNAs layer. By adjusting a curvation correction factor, a debend of the In layer was obtained to make it straight.

For 3DAP, a needle-shaped specimen with a tip radius of $100 \mathrm{~nm}$ was prepared using a FIB-SEM Zeiss NVision40. For details of the preparation method see Thompson et al. (2007).

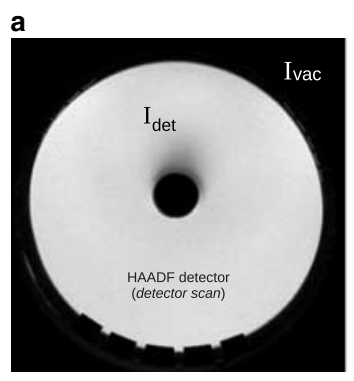

b

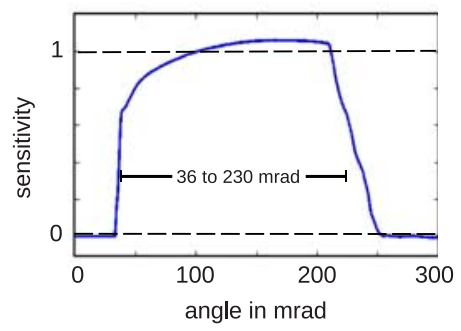

Figure 1. a: Detector scan: image of the HAADF detector in logarithmic scale. b: Sensitivity curve: sensitivity of the detector (radial average).
Before milling operations, the top surface of the wafer was protected by a 100-nm sputter-deposited silicon layer. A 200-nm platinum layer was also deposited using the $30-\mathrm{kV}-\mathrm{Ga}$ beam of the FIB to prevent degradation during the rough milling process. To clean the specimen and minimize ion beam damages, an 1-kV-Ga beam with low incidence angles was used at the end of the milling process.

Figure 2 shows a small part $\left(15 \times 15 \times 50 \mathrm{~nm}^{3}\right)$ of the reconstructed volume related to the $3 \mathrm{DAP}$ analysis of the as-grown sample B. Only indium (pink) and gallium (blue) atoms are shown. The quantum well can be seen as a continuous thin film formed by the indium atoms.

A mass spectrum related to the volume of reconstruction is given in Figure 3. For this particular data set, the noise floor was estimated in a region close to the $\mathrm{N}$ peak at 20 atomic ppm/atom mass unit. Gallium and indium were mainly field evaporated as single charged ions. Numerous molecular ions such as $N_{2}^{+}, A s_{3}^{+}, A s_{3}^{++}$, and $A s_{2}^{+}$were detected. However, an overlap between the $A s_{3}^{++}$and $\mathrm{In}^{+}$ peaks appears in the mass spectrum. Therefore, corrections based on isotope ratio distribution were performed on concentration profiles.

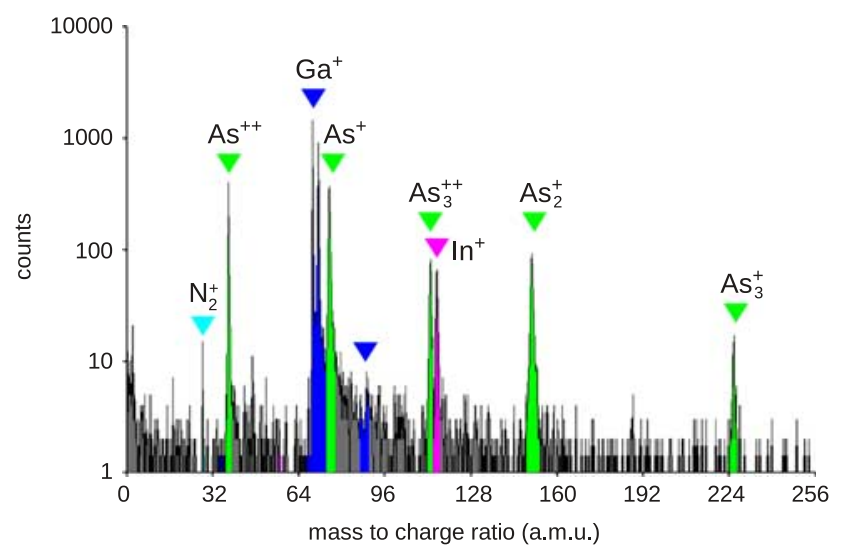

Figure 3. Mass spectrum for the as-grown sample B: number of detected atoms over the mass to charge ratio.

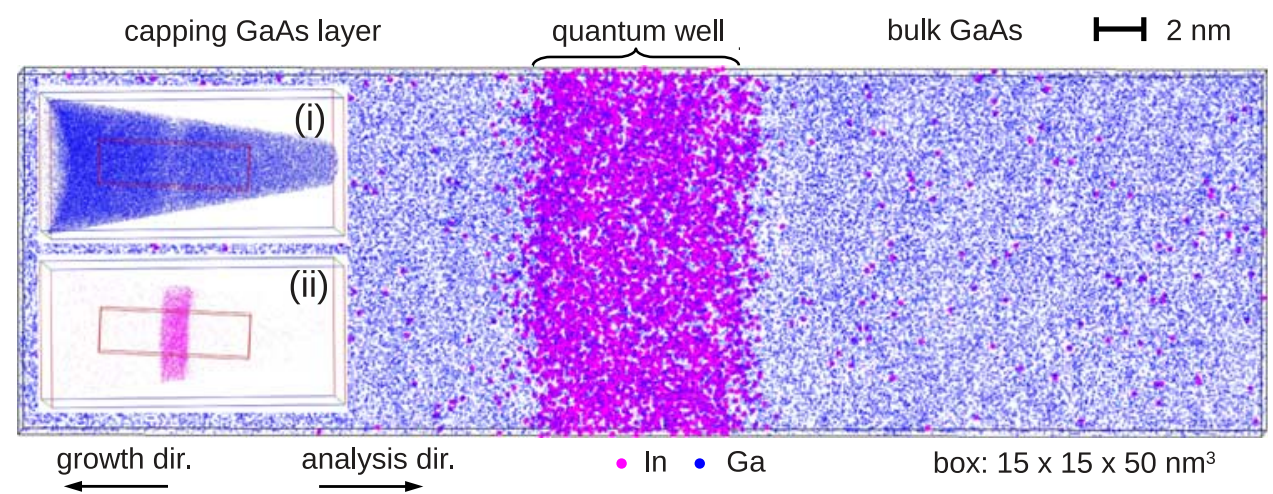

Figure 2. Small part of the reconstructed volume from 3DAP analysis (as-grown sample B), where only indium (pink) and gallium (blue) atoms are shown. Indium atoms form a continuous quantum well. The insets (i, ii) show an extended region of the reconstructed volume with a size of $46 \times 46 \times 98 \mathrm{~nm}^{3}$ for (i) gallium and (ii) indium. 


\section{Reference Data Sets for HAADF-STEM EVALUATION}

For the analysis presented below, two types of information are extracted from the image: the local distances between atomic columns visible in the high-resolution pattern, and the local HAADF intensities. In order to compare these experimental quantities to theoretical ones, two sets of reference data were created containing the theoretical strain and the simulated HAADF intensity as a function of the indium and the nitrogen concentration as well as a function of specimen thickness.

\section{Reference: Strain}

The lattice parameter of the $\operatorname{In}_{x} \mathrm{Ga}_{1-x} \mathrm{~N}_{\mathrm{y}} \mathrm{As}_{1-y}$ unit cell depends on the indium and nitrogen concentrations $x$ and $y$, respectively. Based on the binary lattice constants and on the elastic constants shown in Table 1, the constants of the quaternary system were calculated by a linear combination. The quaternary quantity $A_{\text {InGaNAs }}(x, y)$ is determined from the binary quantities (e.g., $\left.A_{\mathrm{GaN}}\right)$ as follows:

$$
\begin{aligned}
& A_{\mathrm{InGaNAs}}(x, y)=x y \cdot A_{\mathrm{InN}}+x(1-y) \cdot A_{\mathrm{InAs}} . \\
& +y(1-x) \cdot A_{\mathrm{GaN}}+(1-x)(1-y) \cdot A_{\mathrm{GaAs}}
\end{aligned}
$$

HRXRD measurements and TEM investigations showed that the InGaNAs quantum wells were pseudomorphically grown on GaAs. Owing to the high thickness (see Determination of the Local Specimen Thickness section) of the prepared TEM lamellae, a relaxation of the crystal in the electron-beam direction was neglected. As a consequence, the $\mathrm{In}_{x} \mathrm{Ga}_{1-x} \mathrm{~N}_{\mathrm{y}} \mathrm{As}_{1-y}$ unit cell is biaxially strained with tetragonal distortion. The distorted lattice constant $a^{\prime}(x, y)$ of InGaNAs along [001] depends on the bulk-lattice constants $a(x, y)$ of InGaNAs and $a(0,0)=a_{\mathrm{GaAs}}$ for GaAs (substrate) and the elastic constants $c_{11}$ and $c_{12}$ for InGaNAs:

$$
a^{\prime}(x, y)=(1+\varepsilon) \cdot a(x, y),
$$

with

$$
\varepsilon=-2 \frac{c_{12}(x, y)}{c_{11}(x, y)} \cdot \frac{a_{\mathrm{GaAs}}-a(x, y)}{a(x, y)},
$$

derived from Hooke's law.

A reference table was created for the InGaNAs lattice constant in the growth direction that contains the relative InGaNAs lattice constant:

$$
d_{s, n}\left(x_{s}, y_{s}\right)=\frac{a^{\prime}\left(x_{s}, y_{s}\right)}{a_{\mathrm{GaAs}}},
$$

as a function of the concentration values $x_{s}$ and $y_{s}$ :

$$
\begin{aligned}
& x_{s}=[0, \ldots, 0.4] \quad \text { in steps of } 0.02 \\
& y_{s}=[0, \ldots, 0.08] \text { in steps of } 0.01
\end{aligned} .
$$

The index $s$ indicates a quantity obtained by simulation, whereas the index $n$ shows that the quantity is normalized to a reference value [e.g., the lattice constant of GaAs in equation (4)]. This subscription is used in the whole paper.

\section{Reference: HAADF Intensity}

The HAADF intensity of InGaNAs was simulated using the multislice algorithm in the STEMsim (Rosenauer \& Schowalter, 2007) program. For every single simulation, a zincblende GaAs supercell was created with dimensions of $5 \times 5$ unit cells in the [001] growth direction, the [010] direction, and the $200 \mathrm{~nm}$ in [100] electron-beam direction. Periodic boundary conditions were applied perpendicular to the electron-beam direction. In all atomic columns, gallium atoms were statistically replaced by indium atoms and arsenic by nitrogen atoms to achieve an $\operatorname{In}_{x} \mathrm{Ga}_{1-x} \mathrm{~N}_{y} \mathrm{As}_{1-y}$ supercell with the indium and nitrogen concentrations $x$ and $y$. The lattice constants in the [100] and [010] directions were set to $a_{\mathrm{GaAs}}$, and the one in the [001] direction to $a^{\prime}(x, y)$, according to equation (2). Atomic form factors were used, as suggested by Weickenmeier \& Kohl (1991), for calculating the atomic potentials. The whole supercell was relaxed using valence force field (VFF) routines implemented in the LAMMPS code (Plimpton, 1995; Schowalter et al., 2012). Total energy and force of the supercell were minimized according to the method suggested by Keating (1966). Keating potentials were calculated based on the lattice constants and elastic constants in Table 1. It was shown by Müller et al. (2010), Müller et al. (2011), and Volz et al. (2008) that the VFF relaxation is an adequate approach to consider the scattering at SADs in conventional TEM simulations of InGaNAs.

Thermal diffuse scattering was taken into account in terms of the frozen-lattice method, which is an adequate approach to describe electron scattering at phonons in crystals (Wang, 1998, 2003; Van Dyck, 2009; Forbes et al., 2010). For this purpose, all atoms were statistically displaced according to a Gaussian with the width of the mean square

\begin{tabular}{|c|c|c|c|c|c|c|c|}
\hline & $a(\AA)$ & $c_{11}(\mathrm{GPa})$ & $c_{12}(\mathrm{GPa})$ & $\operatorname{BIn}\left(\AA^{2}\right)$ & $\mathrm{BGa}\left(\AA^{2}\right)$ & $\mathrm{BN}\left(\AA^{2}\right)$ & BAs $\left(\AA^{2}\right)$ \\
\hline InAs & 6.058 & 83.29 & 45.26 & 1.0636 & - & - & 0.9008 \\
\hline GaAs & 5.653 & 122.1 & 56.6 & - & 0.7296 & - & 0.6199 \\
\hline InN & 4.98 & 187 & 125 & 0.3983 & - & 0.4473 & - \\
\hline $\mathrm{GaN}$ & 4.5 & 293 & 159 & - & 0.2541 & 0.2928 & - \\
\hline
\end{tabular}
displacements $\left\langle u^{2}\right\rangle$, which was calculated by density functional theory (Schowalter et al., 2009). As it was shown by Schowalter et al. (2009), the generalized-gradient approximation (GGA) in DFT typically leads to an overestimation, e.g., of the lattice

Table 1. Lattice Constants $a$ and Elastic Constants $c_{11}$ and $c_{12}$ for Sphalerite-Type Binary Components of InGaNAs Taken From Vurgaftman et al. (2001).

Debye-Waller temperature factors at $300 \mathrm{~K}$ calculated by DFT (Schowalter et al., 2009). 
parameters, whereas the local-density approximation (LDA) leads to an underestimation. For this reason, the mean values of LDA and GGA were used. Table 1 contains the resulting Debye-Waller temperature factors $B$ in the notation $B=8 \pi^{2}\left\langle u^{2}\right\rangle . B$ is given for all atoms in the specific binary crystal. The Debye-Waller factors of the group-III sublattice were assumed to depend only on the chemical composition of the group- $\mathrm{V}$ sublattice (and vice versa). They were linearly interpolated using equation (1).

Identical microscope parameters to those given in Materials and Methods section were applied to simulate an electron probe. One unit cell of the supercell was scanned by setting $20 \times 20$ scan points. After each slice, the intensity into the angle range between 36 and $230 \mathrm{mrad}$ was multiplied with the sensitivity of the HAADF detector (see Fig. 1b) to take the nonuniform sensitivity of the detector into account. The sum over this HAADF intensity was stored for every slice to achieve scattering data dependent on specimen thickness $t$. Finally, the results of all scan points were averaged to obtain a mean HAADF intensity per unit cell. The scattering intensity represents a fraction of the total probe intensity and is therefore unit less and $<1$.

Simulations were done for all combinations of the concentrations $x=(0,0.1,0.2,0.3,0.4)$ and $y=(0,0.02,0.04,0.08)$. Each simulation, corresponding to a combination of $x$ and $y$, was repeated 15 times with different chemical distributions (which implies different SADs) and different statistical (thermal) displacements. Averaging these 15 simulations gives a mean HAADF intensity per unit cell $I_{s}(x, y, t)$ for the $\mathrm{In}_{x} \mathrm{Ga}_{1-x} \mathrm{~N}_{\mathrm{y}} \mathrm{As}_{1-y}$ material.

$I_{s}$ is interpolated on denser grids $\left(x_{s}, y_{s}\right.$, and $\left.t_{s}\right)$ by $3 \mathrm{D}$ cubic fitting. $x_{s}$ and $y_{s}$ values were used as shown in equation (5), the thickness $t_{s}$ was

$$
t_{s}=[0, \ldots, 200] \mathrm{nm} \text { in steps of } 0.5 \mathrm{~nm} .
$$

For the evaluation the normalized intensities $I_{s, n}\left(x_{s}, y_{s}, t_{s}\right)$ are formed according to:

$$
I_{s, n}\left(x_{s}, y_{s}, t_{s}\right)=\frac{I_{s}\left(x_{s}, y_{s}, t_{s}\right)}{I_{s}\left(0,0, t_{s}\right)} .
$$

$I_{s, n}$ are shown in Figure 4 for selected indium and nitrogen concentrations as a function of thickness. It can be seen that both increasing $x$ and $y$ leads to an increase of the intensity ratio and therefore also of the HAADF intensity. Between 50 and $70 \mathrm{~nm}$ the curves are crossing-here a quantitative evaluation is not possible as different concentrations cannot be distinguished by the measured HAADF intensity. Best conditions for the analysis are at high thicknesses, approximately between 100 and $200 \mathrm{~nm}$ : here the chemical contrast is high and the dependence on the specimen thickness is low.

\section{Chemical Analysis of Indium and Nitrogen}

The description of the method to determine the indium and the nitrogen concentration, from an InGaNAs quantum well is demonstrated by means of the HRSTEM image shown

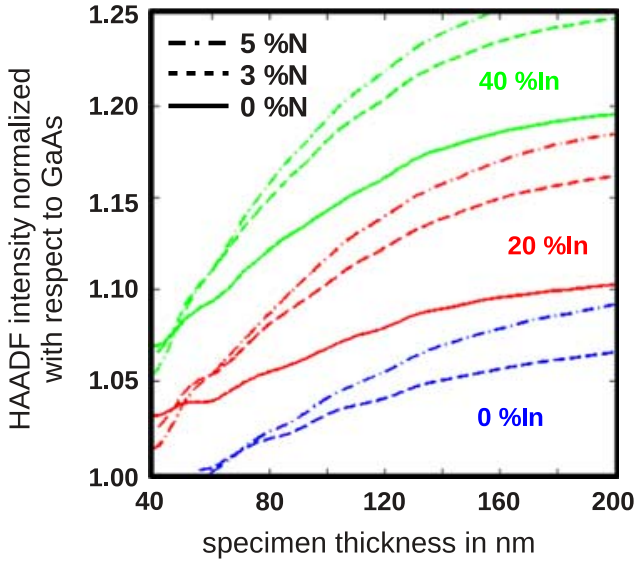

Figure 4. Simulated HAADF intensities for InGaNAs as a function of specimen thickness. Each set of curves (each color) corresponds to a certain indium concentration (from bottom to top: 0,20 , and $40 \%$ indium). Different line styles belong to different nitrogen concentrations (solid: $0 \%$; dashed: $3 \%$; dashed-dotted: $5 \%$ ).

in Figure 5a. It contains a part of the quantum well from sample B (as-grown). The evaluations of sample A and of the annealed sample B were performed in the same way.

\section{Information from the HRSTEM Image}

The smallest scale on which the local strain state can be measured is the distance between two atomic column positions in the HRSTEM image. HAADF contrast within a unit cell depends on the spatial incoherence of the electron beam owing to the source-size effect (Nellist \& Rodenburg, 1994) — whereas the mean intensity per unit cell is unaffected. Owing to these reasons, we evaluated the mean intensity per atom column as described in detail below.

All atomic column positions $P$ (called positions in the following) are found in the noise-filtered HRSTEM image by an iterative routine that allocates the position coordinates to the brightest pixels. For noise filtering, a Wiener and a low pass filter were applied to the original image.

The intensities of all pixels in the unfiltered image whose closest position is the position $j \in P$ were averaged to the mean intensity $I^{\text {raw }}(j) . I^{\text {raw }}(j)$ therefore is the mean value of a patch of pixels around the position $j$. The procedure is called the Voronoi-cell method (see Rosenauer et al., 2011 for details). This is done for all positions $P$ to achieve the mean intensities $I^{\text {raw }}(P)$. As a consequence of the large specimen thickness and the finite probe size, scattering intensity from one scan point (one pixel) stems from a larger lateral region. It is therefore not possible to distinguish between columns of the group-III and the group-V sublattice by comparing the mean intensities per atom column position: all $I^{\mathrm{raw}}(P)$ can therefore be seen as mean intensities per unit cell.

$I^{\text {raw }}(P)$ was normalized with respect to the probe intensity (LeBeau \& Stemmer, 2008; Rosenauer et al., 2009) according to

$$
I(j)=\frac{I^{\mathrm{raw}}(j)-I_{\mathrm{vac}}}{I_{\mathrm{det}}-I_{\mathrm{vac}}} \quad \text { for all } j \in P,
$$




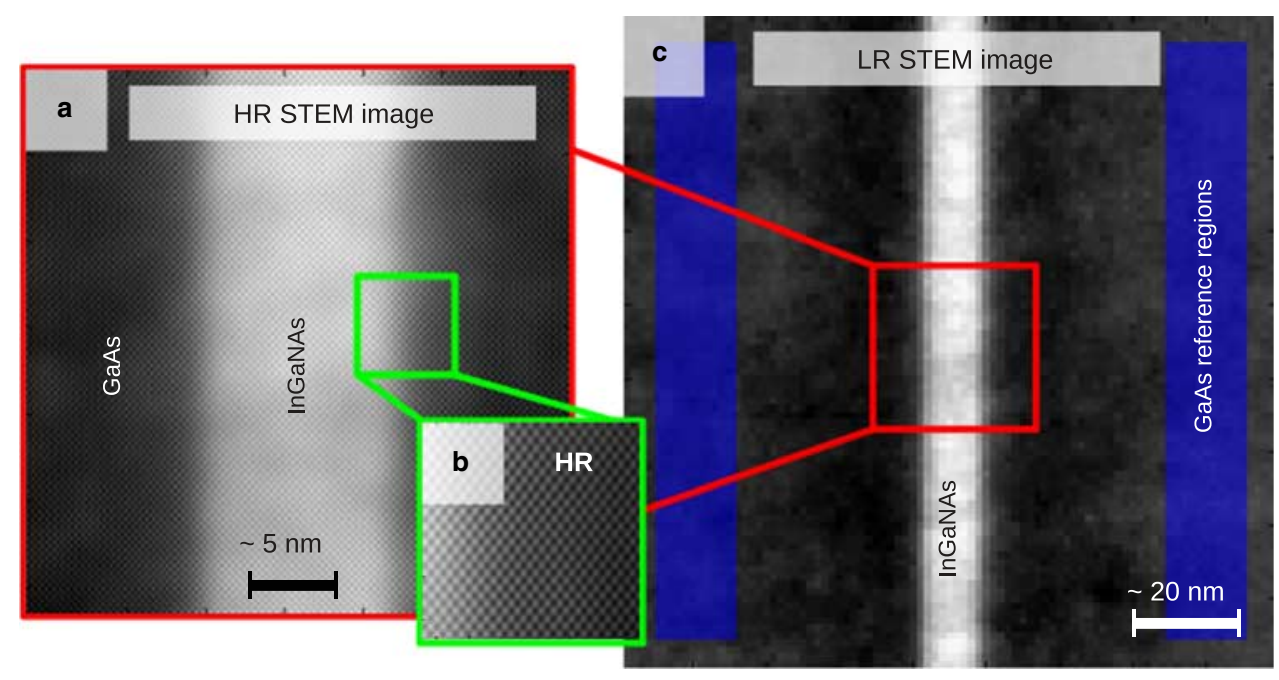

Figure 5. a: The HAADF-HRSTEM image of the as-grown sample B. The image was filtered to detect the atomic column positions that can be seen in the magnification (b). As the GaAs next to the InGaNAs quantum well shows artificially decreased intensity owing to surface strain effects, a larger low-resolution (LR) image (c) was taken at the same position. The GaAs reference regions in (c) contain surface-strain-free GaAs. The thickness was obtained from the unstrained GaAs reference regions of the specimen.

in order to be able to directly compare the measured intensities with the simulated intensities $I_{s}$. $I_{\mathrm{det}}$ and $I_{\mathrm{vac}}$ were measured from the detector scan as explained in Materials and Methods section.

All positions $P$ were determined with subpixel accuracy using a fitting procedure (Grieb et al., 2012), which is not affected by fly-back errors leading to shifted pixel rows caused by the microscope's deflection system. $d(j)$ is the distance in growth direction between two adjacent subpixel positions $j \in P$ and $k \in P$. In general, it is necessary to choose the scan direction so that the fast scan direction is parallel to the direction along which strain is to be measured.

A problem for imaging highly strained quantum wells (owing to the high amount of indium) is the contrast modification of the HAADF intensity owing to effects of surface strain fields. Surface strain fields occur when the material relaxes along the lamella normal owing to the strained quantum well region. This leads to bending of the lattice planes parallel to the electron-beam direction, which causes dechanneling, resulting in an artificial decrease of the HAADF intensity (Grillo, 2009). The GaAs substrate is affected up to a distance of $\sim 20 \mathrm{~nm}$ away from the quantum well. The InGaNAs itself is less affected, as will be shown below, so that a quantitative evaluation is still possible. Owing to this reason, we do not go into detail on the surface strain fields, instead the reader is referred to Grillo (2009), Grillo \& Rossi (2011), and Grieb et al. (2013).

For determination of the specimen thickness presented below a GaAs background free of surface strain artifacts is needed. Therefore, a second image with lower magnification (shown in Fig. 5c) was taken, including the region depicted in Figure 5a. An area of $1 \times 1 \mathrm{~nm}^{2}$ is averaged to achieve a low-resolution (LR) image containing mean HAADF intensities. Two regions in this low-resolution scanning transmission electron microscopy image are defined where unstrained GaAs substrate can be assumed (marked with boxes in Fig. 5c). Based on the intensities in these regions, a $2 \mathrm{D}$ linear polynomial is fitted so that the GaAs intensity is interpolated on the whole image. Finally, the part which would belong to the area of the HR image (marked by a square in Fig. 5c) is cut out and the GaAs intensities are allocated to all positions $P$. The resulting intensities $I_{\mathrm{GaAs}}(P)$ can be seen as the GaAs intensity taking linear thickness variations into account.

By defining a region $U \subset P$ in the GaAs of the highresolution image (not shown in Fig. 5a), the mean atomic distance in growth direction $d_{\mathrm{GaAs}}$ within the GaAs is determined by averaging the local distances in this region: $d_{\mathrm{GaAs}}=\langle d(U)\rangle$. This is possible as surface strain fields have a high influence on the HAADF intensity but less effect on the measurement of strain (Grieb et al., 2012).

The normalized intensities $I_{n}(P)$ are created by dividing all $I(P)$ by the GaAs intensities, $I_{\mathrm{GaAs}}(P)$ and normalized distances $d_{n}(P)$ are created by dividing all $d(P)$ by the GaAslattice distance $d_{\mathrm{GaAs}}$ :

$$
I_{n}(j)=\frac{I(j)}{I_{\mathrm{GaAs}}(j)}, \quad d_{n}(j)=\frac{d(j)}{d_{\mathrm{GaAs}}} \quad \text { for all } j \in P .
$$

\section{Determination of the Local Specimen Thickness}

Rosenauer et al. (2009) demonstrated that a determination of the local specimen thickness within an STEM image is possible by a direct comparison of the HAADF intensity from the substrate region with thickness-dependent simulations of the HAADF intensities. For GaAs, the accuracy was about 5-10 nm and dependent on thickness (Grieb et al., 2012). 

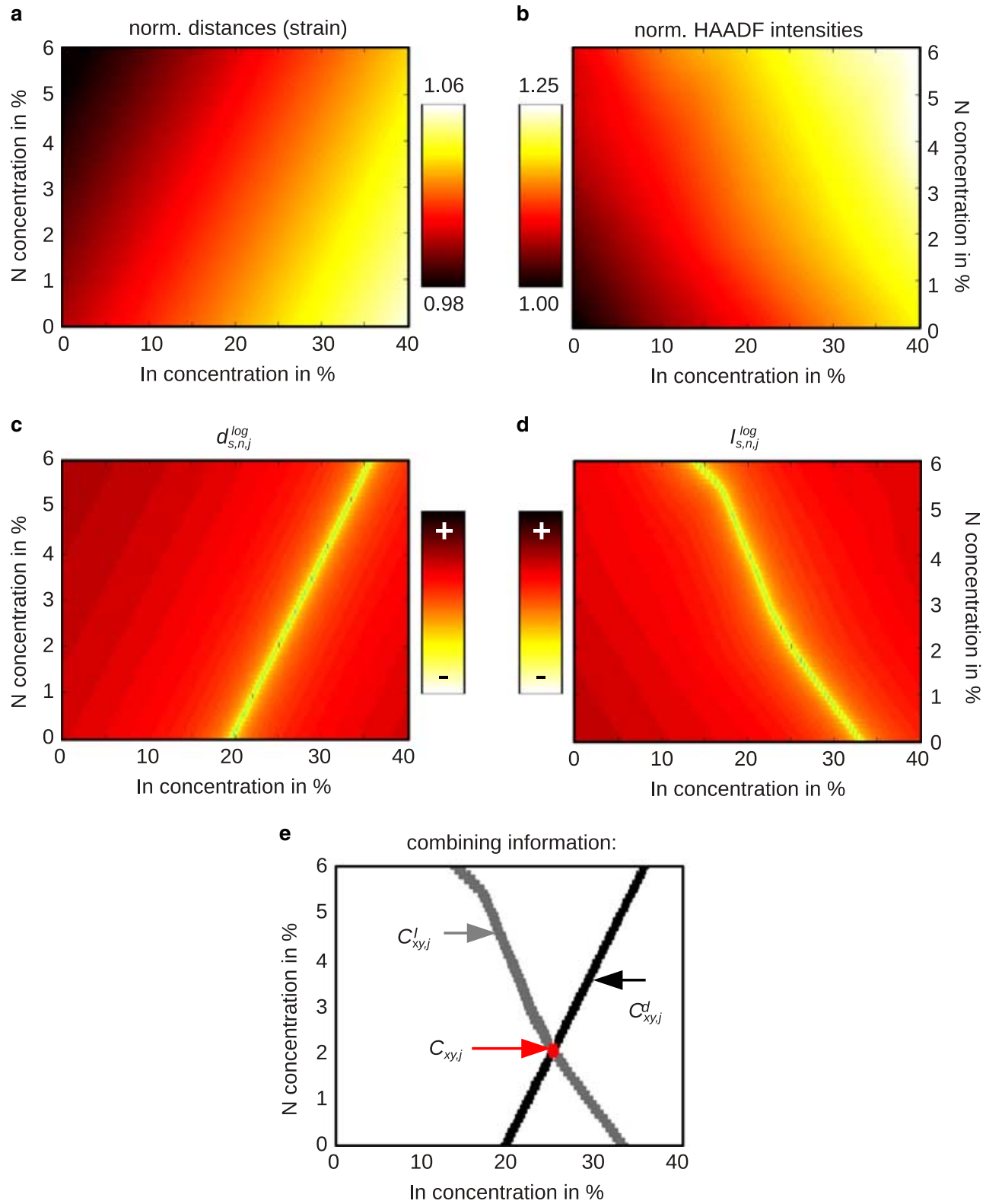

Figure 6. Reference maps as a function of indium and nitrogen concentration for a single position $j \in P$ with (exemplary) measured values of $I_{n}(j)=1.153, d_{n}(j)=1.027$, and $t(j)=150 \mathrm{~nm}$. a: Strain $d_{s, n}$ according to equation (2). b: Simulated HAADF intensities $I_{s, n}$ for $t_{s}=150 \mathrm{~nm}$ normalized with respect to GaAs. (c, d): Bright regions show possible concentration pairs for local strain $d_{n}(j)$ and for local intensity $I_{n}(j)$. e: When combining both types of information from (c) and (d), only concentration pairs $C_{x y, j}$ for a given $d_{n}(j)$ and $I_{n}(j)$ are allowed. The concentrations $C_{x}(j)$ and $C_{y}(j)$ at position $j$ are determined from averaging all pairs $\left(x_{s}, y_{s}\right) \in C_{x y, j}$.

For this reason, the GaAs intensities $I_{\mathrm{GaAs}}(P)$ are compared with the simulated intensities $I_{s}\left(0,0, t_{s}\right)$ for GaAs. For each position $j \in P$ the local thickness $t(j)$ was derived. The mean thickness of the region was $184 \mathrm{~nm}$, where the thickness variation over the image was in the range of the accuracy of the measurement.

There are a few reasons why the measurements were performed at lamellae with comparatively high thicknesses: (1) As shown in Figure 4, the material contrast is high for specimen thicknesses larger than $120 \mathrm{~nm}$. (2) The slope of the signal is smaller for high thicknesses that makes the evaluation less sensitive to errors of the measured thickness. (3) A high thickness is needed to have a significant amount of nitrogen in each group- $\mathrm{V}$ column (statistically 1 nitrogen atom was found in 38-nm thickness). (4) At high thicknesses the strain state of the quantum well can be described by a tetragonal distortion, which simplifies the SSA so simulations of the relaxation state are not necessary.

\section{The Method}

Each atom column position is handled separately. For a certain position $j \in P$ the following information is known: the local 
thickness $t(j)$, the local strain $d_{n}(j)$, and the local (normalized) HAADF intensity $I_{n}(j)$.

The reference data cube for the HAADF intensity is reduced to a $2 \mathrm{D}$ reference map $I_{s, n}\left(x_{s}, y_{s}, t(j)\right)=I_{s, n}\left(x_{s}, y_{s}\right)$ as the local thickness is known. Both reference maps $I_{s, n}$ and $d_{s, n}$ are extrapolated to negative indium and nitrogen concentrations to avoid an artificial biasing of the concentrations owing to noise. Figures $6 \mathrm{a}$ and $6 \mathrm{~b}$ show both reference sets for a thickness of $t(j)=150 \mathrm{~nm}$, where the extrapolated parts are not shown. The HAADF-intensity map shows isolines that are not parallel to the isolines of the strain and therefore allows for unambiguously determining $x$ and $y$. In both reference maps the isolines are found that belong to the local intensity $I_{n}(j)$ and the local strain $d_{n}(j)$, respectively. A good way to find these regions is (exemplarily for the strain) to calculate the map $d_{s, n, j}^{\log }=\log \left(\left|d_{s, n}-d_{n}(j)\right|\right)$. This map is shown in Figure 6c, where the isoline for $d_{n}(j)$ is in the center of the bright valley (minima), visible in the map. Possible concentration pairs $C_{x y, j}^{d}$ for the given strain $d_{n}(j)$ are defined as those for which $d_{s, n, j}^{\text {tog }}\left(x_{s}, y_{s}\right) \leq \sigma$. The threshold $\sigma$ defines the width of the bright valleys shown in Figure 6c and therefore the number of concentration pairs. A threshold $\sigma=\left(2.5 \cdot \operatorname{mean}\left\{d_{s, n, j}^{\log }\right\}-\max \left\{d_{s, n, j}^{\log }\right\}\right)$ was used, but slight deviations from this threshold do not affect the evaluation. Possible concentration pairs $C_{x y, j}^{I}$ were found in the same way for the given HAADF intensity $I_{n}(j)$ (map $I_{s, n, j}^{\log }$ shown in Fig. 6d).

As shown in Figure 6e, $C_{x y, j}$ is the set of all overlapping $C_{x y, j}^{I}$ and $C_{x y, j}^{d}$. Finally, the local indium and nitrogen concentrations $C_{x}(j)$ and $C_{y}(j)$ at the position $j$ are determined from averaging all pairs $\left(x_{s}, y_{s}\right) \in C_{x y, j}$. In the case of the example in Figure $6 C_{x}(j)=0.25$ and $C_{y}(j)=0.02$.

Performing this procedure for all atom-column positions, an indium map $C_{x}(P)$ and a nitrogen map $C_{y}(P)$ are achieved, containing the local concentrations of indium or nitrogen at the positions $P$.

\section{Results ANd Discussion}

\section{Sample A: Low Indium Content}

First, sample A with nominal concentrations of $8 \%$ indium and 3\% nitrogen (from HRXRD) was investigated. A sample of the same growth series was investigated by Müller et al. (2011) and the nominal values could be validated. Because this sample is grown lattice matched to GaAs, no artifacts occurred due to surface-strain fields.

Figure 7 shows the concentration maps and profiles from the HAADF-STEM evaluation by the method presented above. On average, $8.5 \pm 0.9 \%$ of indium and $2.5 \pm 0.3 \%$ of nitrogen are measured that are in good agreement with the nominal values within the accuracy of the method.

To further validate the simulations, a wedge-shaped specimen was prepared using FIB to achieve a thickness gradient between $\sim 40$ and $190 \mathrm{~nm}$.

A large-scale HAADF-STEM intensity map as shown in Figure 8a was acquired: in the center (horizontally) the quantum well can be seen, embedded in GaAs, and no surface strain-induced artifacts are visible. The intensity was normalized according to equation (8) to allow a comparison with simulations.

From the GaAs regions in Figure 8a, the GaAs intensity was obtained and interpolated on the quantum well region as shown in Figure $8 \mathrm{~b}$. By comparing GaAs intensities with $I_{s}\left(0,0, t_{s}\right)$ the thickness was obtained.

In Figure 8c, the measured HAADF intensity is shown as a function of this measured thickness (data points). The straight curves show the simulated intensities for $\mathrm{In}_{0.08} \mathrm{Ga}_{0.92} \mathrm{~N}_{0.03} \mathrm{As}_{0.97}, \mathrm{GaN}_{0.03} \mathrm{As}_{0.97}, \mathrm{In}_{0.08} \mathrm{Ga}_{0.92}, \mathrm{As}$, and $\mathrm{GaAs}$, with and without simulating the effect of SADs.

One can see that the simulations for $\operatorname{In}_{0.08} \mathrm{Ga}_{0.92} \mathrm{~N}_{0.03} \mathrm{As}_{0.97}$ taking SADs into account describe the experimental data well, whereas simulations that ignore the effects of SADs do not. Further curves show that the intensities of the

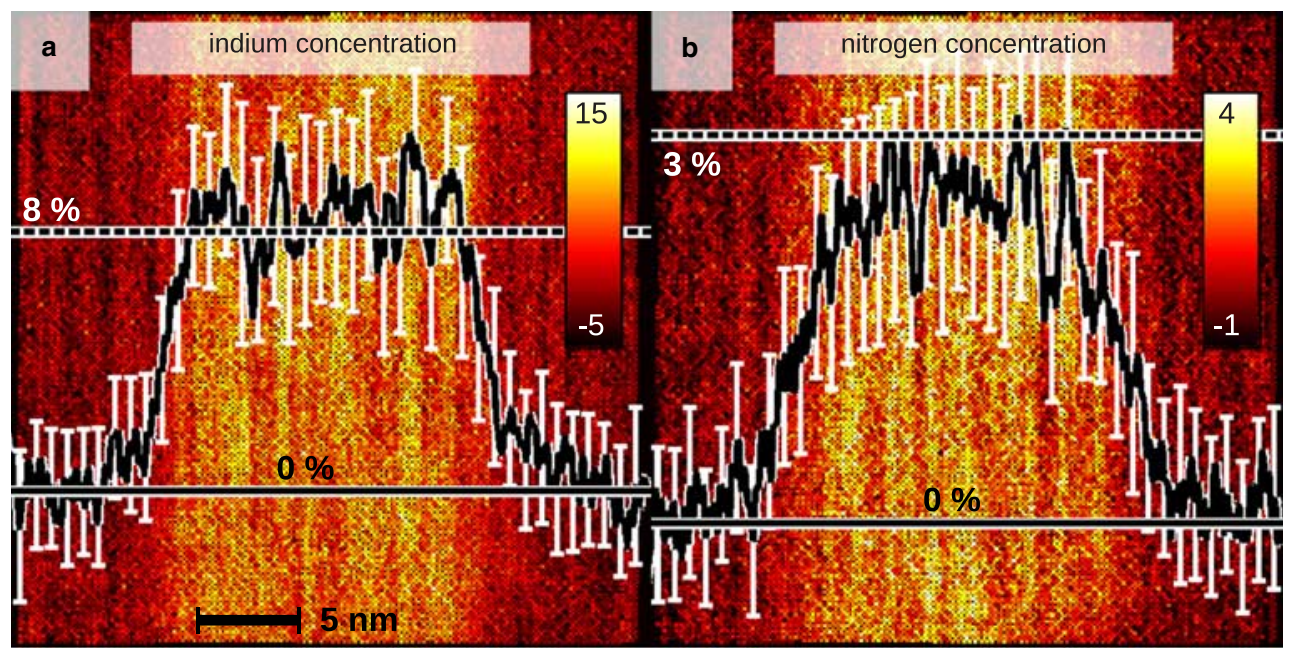

Figure 7. Concentration maps and profiles from high-angle annular dark-field intensity evaluation for the sample A for (a) indium and (b) nitrogen. Horizontal lines mark $0 \%$ and nominal values from HRXRD (8\% indium and 3\% nitrogen). 
a

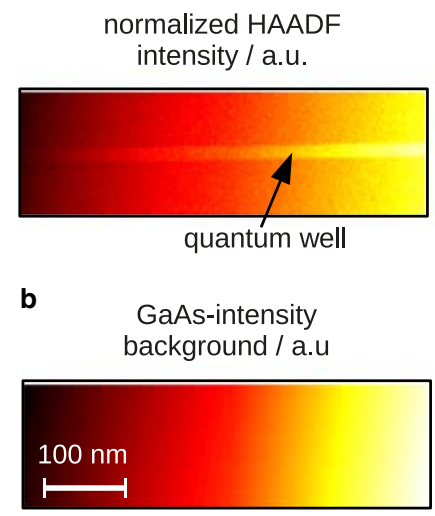

[010]

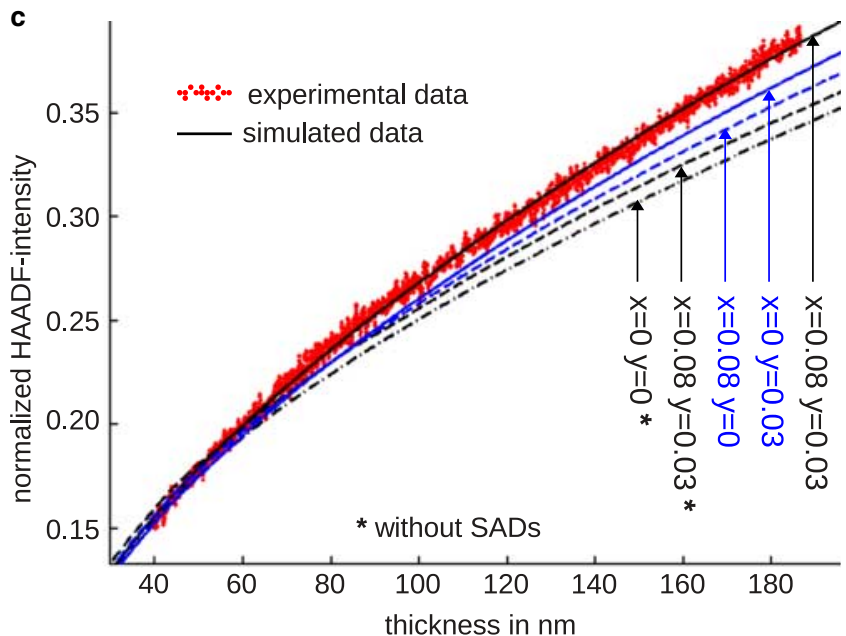

Figure 8. Data obtained from a wedge-shaped sample with low indium concentration (sample A). a: HAADF-intensity map with a large field of view. The quantum well lays horizontally in the center of the map. $\mathbf{b}$ : Background intensities obtained from the GaAs regions in (a), interpolated to the whole map. From this background map a thickness map was determined by a comparison with simulations. c: HAADF intensities (mean intensity per unit cell) as a function of specimen thickness: experimental data (data points) and simulations (lines, from top to bottom: $\operatorname{In}_{0.08} \mathrm{Ga}_{0.92} \mathrm{~N}_{0.03} \mathrm{As}_{0.97}$, $\mathrm{GaN}_{0.03} \mathrm{As}_{0.97}, \mathrm{In}_{0.08} \mathrm{Ga}_{0.92} \mathrm{As}$ (all with SADs), $\mathrm{In}_{0.08} \mathrm{Ga}_{0.92} \mathrm{~N}_{0.03} \mathrm{As}_{0.97}$ (no SADs) and pure GaAs).

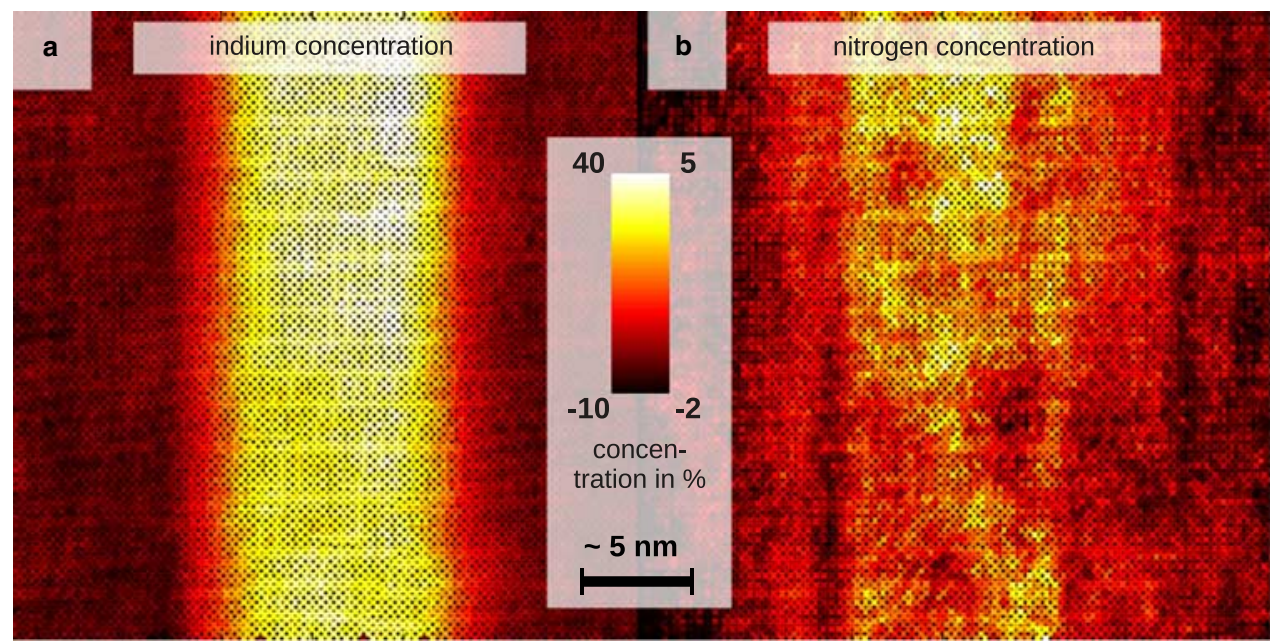

Figure 9. Concentration maps obtained from the HAADF-STEM analysis for the as-grown sample: (a) indium concentration, (b) nitrogen concentration.

ternary $\operatorname{In}_{0.08} \mathrm{GaAs}$ (or $\mathrm{GaN}_{0.03} \mathrm{As}$ ) differ strongly from the experimental data.

\section{Sample B: High Indium Content}

Figure 9 shows the concentration maps obtained (a) for the indium and (b) for the nitrogen concentration for the as-grown sample. In both maps, the quantum-well region is clearly visible by the higher concentrations. Based on these maps, concentration profiles were obtained by averaging the maps perpendicular to growth direction. Figure 10a shows the averaged profiles for the indium concentration and the nitrogen concentration. Gray regions behind both curves show standard deviations calculated when averaging the maps.
Both profiles show negative concentrations in the GaAs region on both sides of the quantum well. This is an artifact owing to the surface strain fields in the vicinity of the quantum well mentioned before. To be able to correctly interpret the concentrations within the quantum well, we determined the specimen thickness from a second STEM image as explained above.

The nominal concentrations from HRXRD measurements are $31 \%$ indium and $1.5 \%$ nitrogen. These values are marked by arrows in Figure 10a. It can be seen that the HAADF-STEM evaluation gives results that are in good agreement with the results from HRXRD (32\% indium and $2 \%$ nitrogen in the center of the well).

The accuracy of the presented technique is limited as both methods, SSA and HAADF-intensity evaluation, are 

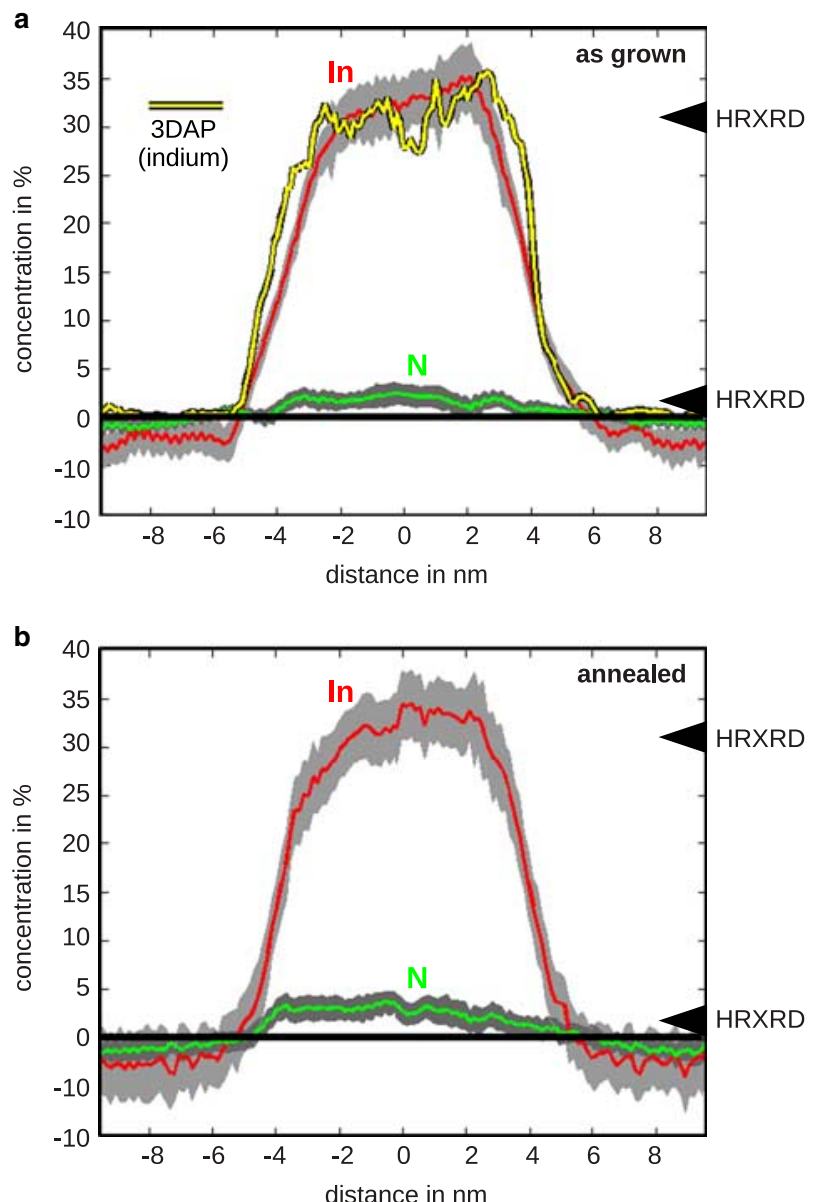

Figure 10. Concentration profiles (a) for the as-grown sample and (b) for the annealed one. Red and green curves show indium and nitrogen profiles from averaging the concentration map perpendicular to the growth direction. Gray regions show standard deviation from averaging. Negative concentration values in the $\mathrm{GaAs}$ besides the quantum well can be explained by intensity artifacts owing to surface strain fields. Values obtained from HRXRD analysis are marked with arrows (upper: In, lower: N). The yellow-black curve in (a) shows the indium-concentration profile obtained from 3DAP measurement.

sensitive to the imaging process and the quality of the highresolution image (affected by noise, high thicknesses, or surface conditions of the specimen). For both individual methods it was shown that they obtain correct concentrations (see, e.g., Rosenauer et al., 2009, 2011; Grieb et al., 2012) but show a large spread over a full STEM image.

The lateral resolution of the method is worse than atomic distances (minimum distance $0.2 \mathrm{~nm}$ ), as cross-talking effects and beam broadening occur.

An HAADF-STEM simulation of a GaAs/InAs interface was performed to estimate the possible lateral resolution. Figure 11 shows the normalized mean intensities for specimen thicknesses of 30,65, and $100 \mathrm{~nm}$ versus distance in units of lattice-plane distances. The interface becomes broader with higher thickness, but the additional broadening increases only slightly with thickness. For the investigated samples, the lateral resolution is expected to be worse than 3 atomic

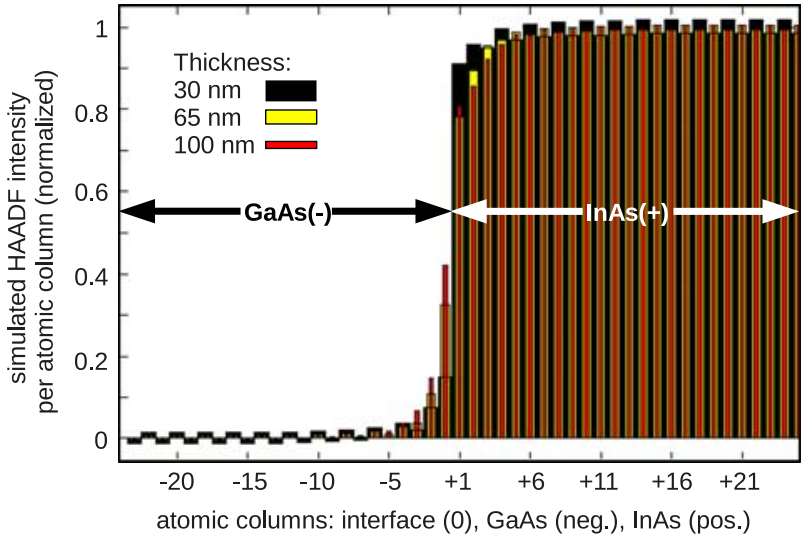

Figure 11. Simulated normalized HAADF-intensity averaged over atomic columns at the interface between pure GaAs (0) and pure InAs (1). The interface becomes less sharp owing to beam broadening. SADs and random variations are not included.

distances $(0.4 \mathrm{~nm})$ as SADs and random variations are not included in the simulation.

The third curve ("3DAP") in Figure 10a shows the indium profile obtained from a 3DAP analysis. For verification, two 3DAP measurements were performed at different positions of the sample, showing comparable results. The mean concentration within the quantum well $(30 \pm 1 \%)$ corresponds to the results from HAADF-STEM and HRXRD. The concentration gradient in the quantum well, measured also by HAADF-STEM analysis, is clearly reproduced. The width of the quantum well in the 3DAP profile is slightly larger in comparison with the HAADF-STEM profile. Here 3DAP is more reliable as the HAADF result is influenced at the InGaNAs/GaAs interfaces by the effects of surface strain fields and beam broadening within the specimen. Nevertheless, it can be seen that both the 3DAP and the HAADF-STEM profiles show same characteristics.

The nitrogen concentration measured by $3 \mathrm{DAP}$ is not shown in Figure 10a. Approximately $0.46 \pm 0.14 \% \mathrm{~N}$ were obtained in the quantum well region, where HAADF-STEM measures $2 \%$, which is in agreement with the results obtained from HRXRD. The missing nitrogen atoms in the 3DAP experiment can be explained by the specific tendency of $\mathrm{N}$ to form molecular ions with other atoms during field evaporation. The existence of $\mathrm{N}+\mathrm{N}$ and $\mathrm{Ga}+\mathrm{N}$ molecular ions have been reported (Saxey, 2011; Riley et al., 2012). One explanation for measuring less nitrogen could be that such molecular ions could be dissociated during flight leading to the formation of neutral N, which would not be detected by the detection system (Saxey, 2011). Another reason could be the preferential evaporation of $\mathrm{N}$ at the low field exerted by the $\mathrm{DC}$ voltage during the intervals between superposing laser pulses. In this case, $\mathrm{N}$ is not detected by time-of-flight mass spectrometry.

Furthermore, HRTEM analyses of the chemical composition was performed using the 3-beam technique as suggested by Müller et al. (2011). With a special L-shaped objective aperture the 000,002 , and 022 beams were selected for image formation. By Fourier filtering 002-fringe amplitude 


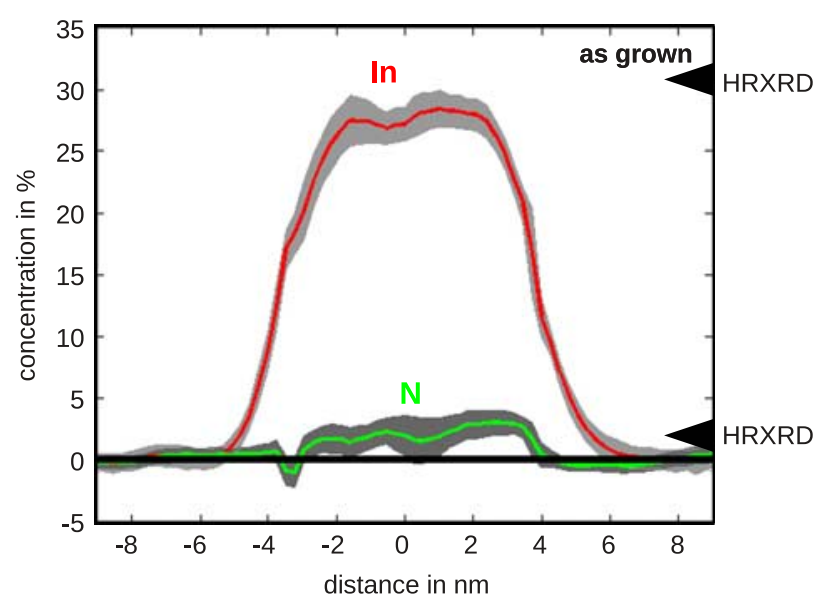

Figure 12. Indium- and nitrogen-concentration profiles obtained from HRTEM-3-beam analysis for the as-grown sample.

as well as the 022 -fringe distances $D_{022}$ were independently extracted from the HRTEM image. The amplitude ratio was normalized to the GaAs signal, which was interpolated to the entire image. This normalized ratio $a_{N}$ strongly depends on the local chemical composition as for zincblende crystals the 002 beam shows chemical sensitivity. The local strain $\varepsilon_{001}$ in the growth direction was determined from the 001 projection of the distances $D_{022}$ normalized to the distances in GaAs.

As shown by Müller et al. (2011) $a_{N}$ can be compared with Bloch-wave simulations taking SADs and bonding effects (MASAs, Rosenauer et al., 2005) into account to get possible combinations for indium and nitrogen concentrations. Here the specimen thickness was $\sim 35 \mathrm{~nm}$. The strain was compared with theoretical values calculated by elasticity theory for different indium and nitrogen concentrations. In combination, $a_{N}$ and $\varepsilon_{001}$ allow determination of the chemical composition at every point in the image.

Figure 12 shows concentration profiles from averaging those composition maps for indium and nitrogen. The gray areas behind the curves show standard deviations from the average. Regions at the quantum-well interface can feature artifacts induced by the analysis as the 002 beam receives a chemical phase jump of $\pi$ at an indium concentration of $\sim 12 \%$ (Müller et al., 2011). The center part of the profile is not influenced by this effect.

The analysis gives concentrations of $28 \pm 2 \%$ indium and $1.5 \pm 1.5 \%$ nitrogen. Evaluations were performed at different thicknesses within the range of $35 \pm 15 \mathrm{~nm}$ in order to check how the exact specimen thickness influences the obtained concentrations. Only small deviations in the mean concentrations were observed: the range of concentration difference is $<1 \%$ of indium and $<0.5 \%$ of nitrogen. Within the uncertainty of both measurements, these results are in agreement with those obtained from HAADF-STEM analysis.

\section{Annealing Effects}

The same evaluation of the HAADF-STEM intensity was also performed for the annealed sample B. Figure 10b shows

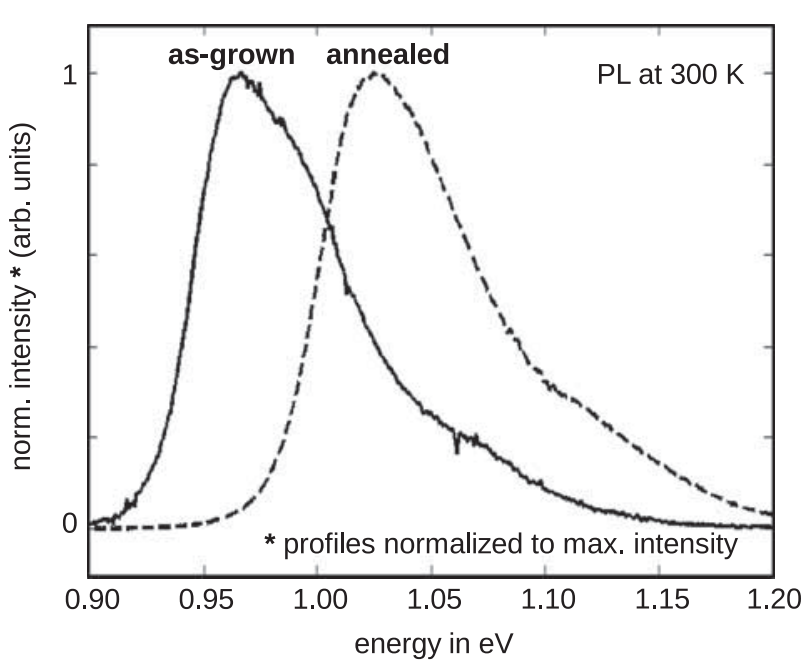

Figure 13. Room temperature photoluminescence data of sample B. The intensity profiles were normalized with respect to their maximum intensity. An annealing-induced energy shift of $60.6 \mathrm{meV}$ from $0.966 \mathrm{eV}$ (as-grown) to $1.025 \mathrm{eV}$ (annealed) was observed.

the concentration profiles for the annealed sample with the identical line style as used in Figure 10a. The indium concentration obtained from HAADF-STEM $(\sim 32 \%)$ is in agreement with the HRXRD results (31\%), where the nitrogen concentration is slightly higher $(\sim 2.7 \%$, HRXRD: $1.5 \%)$. Nevertheless, deviation for the nitrogen concentration is in the range of the accuracy of the evaluation method. Comparing the indium profiles of the annealed and the as-grown sample, differences in the mean indium concentration cannot be observed within the accuracy of the method.

The PL measurements shown in Figure 13 result in a peak maximum at $0.966 \mathrm{eV}$ for the as-grown and $1.025 \mathrm{eV}$ for the annealed sample. The presented concentration measurements give no hints for elemental diffusion or homogenization in a range that would explain an energy shift of $59 \mathrm{meV}$-therefore the observed blue shift must have a different reason. As the energy shift is similar to what was measured by Klar et al. (2001) (a shift of $\sim 70 \mathrm{meV}$ for an $\mathrm{In}_{0.3} \mathrm{Ga}_{0.7} \mathrm{~N}_{0.01} \mathrm{As}_{0.99}$ sample), this leads to the assumption that the observed shift is caused as suggested by Klar et al. (2001) and by Kurtz et al. (2001), namely by a redistribution of the next-neighbor correlation of the elements. The thermal energy allows nitrogen atoms to switch from gallium-rich to indium-rich lattice sites (Klar et al., 2001; Kurtz et al., 2001) ( $N$-hopping) as In-N bonds in the bulk crystal are energetically more favorable (Wagner et al., 2003) compared with $\mathrm{Ga}-\mathrm{N}$ bonds. That this redistribution leads to a reduction of the band gap resulting in a blue shift of the PL spectrum was shown by Kim \& Zunger (2001).

\section{Conclusion and Summary}

HAADF-STEM scattering intensity of InGaNAs can be simulated using a frozen-lattice multislice approach. The influence 
of SADs by relaxing the supercell using VFF routines, as well as the nonhomogeneous sensitivity of the HAADF detector, were taken into account.

We suggest a method to evaluate the indium and the nitrogen concentration of InGaNAs quantum wells simultaneously from a STEM image. For this purpose, two methods of evaluation are combined: the HAADF intensity was compared with simulations and the local lattice distances from the high-resolution STEM image were measured and a SSA was applied. With both types of information, the local HAADF intensity and the local strain, concentration maps and profiles were obtained from two InGaNAs/GaAs quantum well samples A and B.

Sample A with a low indium content (nominal 8\% indium and $3 \%$ nitrogen as used in solar cells) was investigated with the suggested HAADF-STEM analysis: the obtained concentrations are in agreement with HRXRD and TEM-3-beam analysis. By using a wedge-shaped sample it could be shown that the simulations accurately describe the measured intensity for the whole measured thickness range $(40-190 \mathrm{~nm})$. This comparison clearly indicates that scattering at static SADs plays an important role and therefore has to be taken into account for the simulation of the HAADF intensity of InGaNAs.

Sample B with a high indium content (nominal 31\% indium and $1.5 \%$ nitrogen as used in laser diodes) was investigated as-grown and annealed at $725^{\circ} \mathrm{C}$ under As-stabilized conditions. For the as-grown sample we determined the indium concentration to be about $32 \%$ that is in agreement with results from 3D atom probe analysis, HRXRD, and TEM-3-beam analysis. The nitrogen concentration was determined to be about $2 \%$, which also fits well with the results from HRXRD and TEM-3-beam analysis.

Photoluminescence shows an annealing-induced energy shift of $59 \mathrm{meV}$, but we did not observe changes of composition that could explain this shift. Instead, the results can be explained by a model suggested by Klar et al. (2001) and Kurtz et al. (2001), where only an internal redistribution of the nitrogen atoms takes place but the total composition remains constant. It is important to note that quantification of the effect on the HAADF intensity caused by this deviation from a random-alloy composition requires further simulations and is not a subject of this paper.

\section{ACKNOWLEDGMENTS}

This work was supported by the German Research Foundation (DFG, SCHO1196/3-1, RO2057/8-1-3008074, Research Training Group GRK1782).

\section{ReFERENCES}

Albrecht, M., Grillo, V., Remmele, T., Strunk, H.P., Egorov, A.Y., Dumitras, G., Riechert, H., Kaschner, A., Heitz, R. \& Hoffmann, A. (2002). Effect of annealing on the In and $\mathrm{N}$ distribution in InGaAsN quantum wells. Appl Phys Lett 81(15), 2719-2721.
Buyanova, I.A., Pozina, G., Hai, P.N., Thinh, N.Q., Bergman, J.P., Chen, W.M., XIN, H.P. \& Tu, C.W. (2000). Mechanism for rapid thermal annealing improvements in undoped $\mathrm{GaN}_{x} \mathrm{As}_{1-x} / \mathrm{GaAs}$ structures grown by molecular beam epitaxy. Appl Phys Lett 77, 2325-2327.

Carlino, E. (2010). Quantitative Z-contrast atomic resolution studies of semiconductor nanostructured materials. J Phys Conf Ser 209, 012005.

Forbes, B.D., Martin, A.V., Findlay, S.D., D’Alfonso, A.J. \& Allen, L.J. (2010). Quantum mechanical model for phonon excitation in electron diffraction and imaging using a Born-Oppenheimer approximation. Phys Rev B 82, 104103.

Friedman, D.J., Geisz, J.F., KurTz, S.R. \& Olson, J.M. (1998). 1-Ev solar cells with GaInNAs active layer. J Cryst Growth 195, 409-415.

GLAS, F. (2004). The effect of the static atomic displacements on the structure factors of weak reflections in cubic semiconductor alloys. Philos Mag 84(20), 2055-2074.

Grieb, T., Müller, K., Fritz, R., Grillo, V., Schowalter, M., Volz, K. \& Rosenauer, A. (2013). Quantitative chemical evaluation of dilute GaNAs using ADF STEM: Avoiding surface strain induced artifacts. Ultramicroscopy 129, 1-9.

Grieb, T., Müller, K., Fritz, R., Schowalter, M., Neugebohrn, N., Knaub, N., Volz, K. \& Rosenauer, A. (2012). Determination of nitrogen concentration in dilute GaNAs by STEM HAADF Z-contrast imaging and STEM-HAADF strain state analysis. Ultramicroscopy 117, 15-23.

GriLlo, V. (2009). The effect of surface strain relaxation on HAADF imaging. Ultramicroscopy 109, 1453-1464.

Grillo, V., Albrecht, M., Remmele, T., Strunk, H.P., Egorov, A.Y. \& RIECHERT, H. (2001). Simultaneous experimental evaluation of In and $\mathrm{N}$ concentrations in InGaAsN quantum wells. $J$ Appl Phys 90(8), 3792-3798.

Grillo, V., Carlino, E. \& Glas, F. (2008). Influence of the static atomic displacement on atomic resolution $\mathrm{Z}$-contrast imaging. Phys Rev B 77, 054103.

Grillo, V. \& Rossi, F. (2011). A new insight on crystalline strain and defect features by STEM-ADF imaging. J Cryst Growth 318, 1151-1156.

Keating, P.N. (1966). Effect of invariance requirements on the elastic strain energy of crystals with application to the diamond structure. Phys Rev 145(2), 637-645.

KIM, K. \& Zunger, A. (2001). Spatial correlations in GaInAsN alloys and their effects on band-gap enhancement and electron localization. Phys Rev Lett 86, 2609-2612.

Kitatani, T., Nakahara, K., Kondow, M., Uomi, K. \& Tanaka, T. (2000). Mechanism analysis of improved GaInNAs optical properties through thermal annealing. J Cryst Growth 209, 345-349.

Klar, P.J., Grüning, H., Koch, J., Schäfer, S., Volz, K., Stolz, W., Heimbrodt, W., Kamal SaAdi, A.M., Lindsay, A. \& O’Reilly, E.P. (2001). (Ga, In)(N, As)-fine structure of the band gap due to nearest-neighbor configurations of the isovalent nitrogen. Phys Rev B 64(12), 121203.

Kondow, M., Kitatani, T., Nakatsuka, S., Larson, M.C., NaKahara, K., Yazawa, Y., ОкaI, M. \& Uomi, K. (1997). GaInNAs: A novel material for long-wavelength semiconductor lasers. IEEE J Sel Topics Quantum Electron 3, 719-730.

Kurtz, S., Webb, J., Gedvilas, L., Friedmann, D., Geisz, J., Olson, J., King, R., Joslin, D. \& Karam, N. (2001). Structural changes during annealing of GaInAsN. Appl Phys Lett 78, 748-750. 
LeBeau, J. \& Stemmer, S. (2008). Experimental quantification of annular dark-field images in scanning transmission electron microscopy. Ultramicroscopy 108, 1653-1658.

Litvinov, D., Gerthsen, D., Rosenauer, A., Hetterich, M., Grau, A., Gilet, P. \& Grenouillet, L. (2004). Determination of the nitrogen distribution in InGaNAs/GaAs quantum wells by transmission electron microscopy. Appl Phys Lett 85, 3743-3745.

Mehrtens, T., Bley, S., Satyam, P.V. \& Rosenauer, A. (2012). Optimization of the preparation of GaN-based specimens with low-energy ion milling for (S)TEM. Micron 43, 902-909.

Molina, S.I., Sales, D.L., Galindo, P.L., Fuster, D., González, Y., Alén, B., González, L., Varela, M. \& Pennycook, S.J. (2009). Column-by-column compositional mapping by Z-contrast imaging. Ultramicroscopy 109, 172-176.

Müller, K., Schowalter, M., Rosenauer, A., Hu, D., Schaddt, D.M., Hetterich, M., Gilet, P., Rubel, O., Fritz, R. \& Volz, K. (2011). Atomic scale annealing effects of $\operatorname{In}_{x} \mathrm{Ga}_{1-x} \mathrm{~N}_{y} \mathrm{As}_{1-y}$ studied by TEM three-beam imaging. Phys Rev B 84, 045316.

Müller, K., Schowalter, M., Rosenauer, A., Rubel, O. \& Volz, K. (2010). Effect of bonding and static atomic displacements on composition quantification in $\mathrm{In}_{x} \mathrm{Ga}_{1-x} \mathrm{~N}_{y} \mathrm{As}_{1-y}$. Phys Rev $B$ 81(7), 075315.

Nellist, P. \& Rodenburg, J. (1994). Beyond the conventional information limit: The relevant coherence function. Ultramicroscopy 54, 61-74.

Plimpton, S. (1995). Fast parallel algorithms for short-range molecular dynamics. J Comput Phys 117, 1-19.

Riechert, H., Ramakrishnan, A. \& Steinle, G. (2002). Development of InGaAsN-based $1.3 \mu \mathrm{m}$ VCSELs. Semicond Sci Technol 17, 892-897.

Riley, J., Bernal, R., Li, Q., Espinosa, H., WANG, G. \& Lauhon, L. (2012). Atom probe tomography of a-axis GaN nanowires: Analysis of nonstoichiometric evaporation behavior. ACS Nano 6, 3898-3906.

Rosenauer, A., Fischer, U., Gerthsen, D. \& Förster, A. (1998). Composition evaluation by lattice fringe analysis. Ultramicroscopy 72, 121-133.

Rosenauer, A., Gries, K., Müller, K., Pretorius, A., Schowalter, M., Avramescu, A., Engl, K. \& Lutgen, S. (2009). Measurement of specimen thickness and composition in $\mathrm{Al}_{x} \mathrm{Ga}_{1-} \mathrm{N} / \mathrm{GaN}$ using high-angle annular dark field images. Ultramicroscopy 109, 1171-1182.

Rosenauer, A., Mehrtens, T., Müller, K., Gries, K., Schowalter, M., Satyam, P.V., Bley, S., Tessarek, C., Hommel, D., Sebald, K., Seyfried, M., Gutowski, J., Avramescu, A., Engl, K. \& Lutgen, S. (2011). Composition mapping in InGaN by scanning transmission electron microscopy. Ultramicroscopy 111, 1316-1327.

Rosenauer, A. \& Schowalter, M. (2007). STEMSiM - A new software tool for simulation of STEM HAADF Z-contrast imaging. Springer Proc Phys 120, 169-172.
Rosenauer, A., Schowalter, M., Glas, F. \& Lamoen, D. (2005). Firstprinciples calculations of 002 structure factors for electron scattering in strained $\operatorname{In}_{x} \mathrm{Ga}_{1-x}$ As. Phys Rev B 72, 085326.

SAXEY, D. (2011). Correlated ion analysis and the interpretation of atom probe mass spectra. Ultramicroscopy 111, 473-479.

Schowalter, M., Müller, K. \& Rosenauer, A. (2012). Scattering amplitudes and static atomic correction factors for the composition-sensitive 002 reflection in sphalerite ternary iii-v and ii-vi semiconductors. Acta Cryst A 68, 68-76.

Schowalter, M., Rosenauer, A., Titantah, J.T. \& Lamoen, D. (2009). Computation and parametrization of the temperature dependence of Debye-Waller factors for group iv, iii-v and ii-vi semiconductors. Acta Cryst A 65, 5-17.

Scott, J., Docherty, F.T., MacKenzie, M., Smith, W., Miller, B., Coluins, C.L. \& Craven, A.J. (2006). Sample preparation for nanoanalytical electron microscopy using the FIB lift-out method and low energy ion milling. J Phys Conf Ser 26, 223-226.

Spruytte, S.G., Larson, M.C., Wampler, W., Coldren, C.W., Petersen, H.E. \& Harris, J.S. (2001). Nitrogen incorporation in group iii-nitride-arsenide materials grown by elemental source molecular beam epitaxy. J Cryst Growth 227-228, 506-515.

Thompson, K., Lawrence, D., Larson, D.J., Olson, J.D., Kelly, T.F. \& Gorman, B. (2007). In situ site-specific specimen preparation for atom probe tomography. Ultramicroscopy 107, 131-139.

VAN Dyck, D. (2009). Is the frozen phonon model adequate to describe inelastic phonon scattering? Ultramicroscopy 109, 677-682.

Volz, K., Torunski, T., Kunert, B., Rubel, O., Nau, S., Reinhard, S. \& STOLZ, W. (2004). Specific structural and compositional properties of (GaIn)(NAs) and their influence on optoelectronic device performance. J Cryst Growth 272, 739-747.

Volz, K., Torunski, T., Rubel, O. \& Stolz, W. (2008). Direct structural evidence of the change in n-iii bonding in (GaIn) (NAs) before and after thermal annealing. J Appl Phys 104, 053504.

Vurgaftman, I., Meyer, J.R. \& Ram-Mohan, L.R. (2001). Band parameters for iii-v compound semiconductors and their alloys. J Appl Phys 89(11), 5815-5875.

Wagner, J., Geppert, T., KüOhler, K., Ganser, P. \& Maier, M. (2003). Bonding of nitrogen in dilute GaInAsN and AlGaAsN studied by Raman spectroscopy. Solid State Electron 47, 461-465.

WANG, Z. (1998). The "frozen-lattice" approach for incoherent phonon excitation in electron scattering. How accurate is it? Acta Cryst A 54, 460-467.

WANG, Z. (2003). Thermal diffuse scattering in sub-angstrom quantitative electron microscopy - Phenomenon, effects and approaches. Micron 34, 141-155.

Weickenmeier, A. \& KoHL, H. (1991). Computation of absorptive form factors for high-energy electron diffraction. Acta Cryst A 47(5), 590-597. 